\title{
Una pandemia y varios males endémicos en el Perú: A propósito de la COVID-19
}

\section{A pandemic and several endemic ills in Perú: Regarding COVID-19}

\author{
Jaime Salazar-Zuloeta ${ }^{(1, a)}$, Rosío Pando-Lazo ${ }^{(1, b)}$
}

\section{RESUMEN}

El Perú, pese al avance económico alcanzado en las últimas décadas, y luego de anunciar y aprobar reformas, no ha podido hacer efectiva la consolidación de un sólido sistema de salud, capaz de llegar con atenciones oportunas, seguras, efectivas y eficientes, sobre todo, a las poblaciones con menos recursos económicos. Esta deficiencia se deja notar desde el primer nivel de atención, y se agudiza en el defectuoso y burocrático sistema de referencia a otros niveles de complejidad. También es lamentable que la inversión económica en salud no sea la que se requiere para corregir la inequidad. Como si lo anterior no fuera suficiente, sistemas de corrupción funcionando desde hace décadas terminan afectando sentidamente el otorgamiento de atenciones de salud. La inversión en generación de conocimiento y tecnología en salud, por años abandonada, está avanzando gracias a acertadas decisiones como la Ley Marco de Telesalud que ha puesto la tecnología a disposición las clases populares menos favorecidas del país.

Palabras Clave: Inversión para la Salud; Acceso Universal en los Sistemas de Salud; Inequidad en la salud; Servicios de telemedicina.

\section{ABSTRACT}

Despite the economic progress achieved in recent decades, and after announcing and approving reforms, Peru has not been able to make effective the consolidation of a solid Health System, capable of reaching, with timely, safe, effective and efficient care, above all, to populations with fewer economic resources. This deficiency is noted from the first level of attention, and is exacerbated by the flawed and bureaucratic system of reference to other levels of complexity. It is also regrettable that economic investment in health is not what is required to correct inequity. As if the above were not enough, corruption systems that have been operating for decades end up having a profound effect on the provision of health care. Investment in the generation of knowledge and health technology, neglected for years, is advancing thanks to wise decisions such as the Telehealth Framework Law that has made technology available to the less favored popular classes in the country.

Keywords: Investment for health; Universal access to health care services; Health inequity; Telemedicine services.

\footnotetext{
${ }^{1}$ Facultad de Medicina Humana. Universidad Nacional Pedro Ruiz Gallo. Lambayeque, Perú

${ }^{a}$ Médico Infectólogo y Tropicalista. Magister en Ciencias con Mención en Gerencia de Servicios de Salud.

${ }^{\mathrm{b}}$ Médico Anestesiólogo. Auxiliar
} 


\section{INTRODUCCIÓN}

Es innegable que nuestro país, con un sistema de salud frágil y precario, prácticamente ha sucumbido frente a la indetenible demanda de infectados por el SARS-CoV-2; lamentablemente esta no es una realidad solo del Perú, también lo es en varios países de América Latina, nuevo foco de la pandemia, afectando tanto la salud de las personas, así como las frágiles economías de estas naciones (1).

De hecho, los expertos opinan que prácticamente ningún país del mundo podría ser capaz de soportar la embestida de esta pandemia, y sin duda, esto ha quedado demostrado cuando países del primer mundo como Italia llegaron a padecer serios problemas para manejar su propia demanda de pacientes (2). Similar escenario se observa en este momento en el que se considera el país con más poder en el mundo, Estados Unidos, con más de 5 millones de infectados y 165 mil defunciones atribuidas al SARS-CoV-2 ${ }^{(3)}$.

Pero unido al sufrimiento por la morbilidad y mortalidad debida a la pandemia, los autores creemos que es necesario mencionar varios de los que consideramos males endémicos dentro de nuestro país, males que han colocado en cuidados intensivos a prácticamente todo el Perú.

Consideramos que los podemos describir de la siguiente forma:

Sistema de salud fragmentado, desarticulado. En el Perú funcionan, bajo la rectoría del Ministerio de Salud (Minsa), subsistemas fragmentados que, desgraciadamente, no interactúan, trabajan de modo descoordinado. En efecto, las Instituciones Prestadoras de Servicios de Salud (IPRESS) del Minsa ofrecen servicios a la población pobre con seguro público y sin seguro de salud, EsSalud que atiende a trabajadores del sector público y privado y sus familiares; luego vienen los hospitales del Ministerio de Defensa e Interior, que atienden a personal de las Fuerzas Armadas y Policiales, respectivamente; $y$, por último, el sector privado, que ofrece cobertura con aportes de empleadores, hogares y Cooperación Internacional (4). Cada sector trabaja por su cuenta, lleva sus propias estadísticas, genera sus propios documentos técnicos (incluso los que, por norma, son generados y publicados por el Minsa); en suma, no podríamos hablar de un auténtico Sistema de Salud peruano, y eso ha contribuido en parte a la catástrofe nacional respecto al manejo de la covid-19.

A pesar de las intenciones de la Reforma de Salud de 2013, la desarticulación es tan marcada que, ante el evidente colapso de los hospitales del Minsa y EsSalud y frente al pedido del Gobierno de que se establezcan convenios con clínicas privadas para que atiendan casos de la covid-19 a pacientes que cuentan con Seguro Integral de Salud (SIS), solamente 14 de estos establecimientos han respondido al pedido gubernamental ${ }^{(5)}$.
Otra de las consecuencias del trabajo desarticulado es la inequidad en la distribución geográfica de recursos humanos en salud; en efecto, pese al aumento del número nacional de médicos especialistas y enfermeros, de modo que mientras Lima y las regiones del litoral cuentan con las densidades más altas, hay regiones como Piura, Loreto y Lambayeque que aparecen en el extremo de menores asignaciones de personal de salud ${ }^{(4,6)}$.

Sin duda, actualmente estamos lejos de lograr el óptimo y oportuno intercambio prestacional y el usuario, en especial el que está en estado de pobreza y pobreza extrema. Si la idea es que ningún peruano se quede sin recibir atención de salud, pues simplemente esto por ahora no se está produciendo y, en cambio, hoy, en plena efervescencia de la pandemia, se nota que persiste la discriminación e ineficiencias a pesar de las anunciadas reformas en el sector ${ }^{(7)}$.

Como Sistema de Salud, el peruano no es permanentemente evaluado, como si ocurre, por ejemplo, con los sistemas de salud europeos (7).

Por último, como si la desarticulación en el propio sistema de salud no fuera suficiente, la percepción de la ciudadanía, confirmada por la realidad, es que los planes de Salud no coinciden necesariamente con las de otros sectores como Educación, Economía, Vivienda, Agricultura, Minería, entre otros, lo que trae como consecuencia que no podamos dar la talla en el desarrollo integral de la población, aspiración justa que tendría que ser, a nuestro juicio, una de las directrices esenciales del Gobierno

Escaso nivel de fortalecimiento de la Atención Primaria de Salud. Los sistemas de Atención Primaria de Salud (APS) deben apuntar a mejorar las condiciones de vida de las personas y alcanzar la equidad, y deberían orientarse a la promoción de la salud y prevención del daño y de la enfermedad; pero, en el Perú todavía persisten altas las tasas de mortalidad infantil y coberturas inadecuadas en salud materna, solo para citar dos ejemplos de lo que un buen Primer Nivel de Atención debería cautelar haciendo uso de sus recursos y extremando los cuidados. Por años, lo que llamamos atención primaria en salud en el Perú ha recibido un fortalecimiento marginal, no el suficiente como para servir como anillo de contención para el manejo de la patología no compleja y el despliegue de las políticas nacionales de Salud, por ejemplo, las inmunizaciones; un buen sistema de atención primaria debería tener la capacidad de llegar hasta el "último rincón de la patria", con una atención eficaz, eficiente, segura y oportuna. De hecho, el espíritu de la Declaración de Alma-Ata en Rusia, entre el 6 y 12 de se- 
tiembre de 1978, fue tener "salud para todos en el año 2000", bajo el principio "la promoción y prevención de la salud de los pueblos es indispensable para un desarrollo social y económico sostenido", anhelo que, en honor a la verdad, no hemos cumplido a cabalidad (8).

Aun con sus falencias, es el Minsa el único que tiene la capacidad de llegar hasta los lugares más remotos del Perú, incluyendo a los pequeños pueblos asentados a orillas del Amazonas; sin embargo, aún quedan brechas por cerrar entre lo que es y lo que debería ser considerado como un nivel adecuado de atención, como cuando nos llegaba el grito de auxilio de los líderes de las comunidades indígenas frente al avance de la covid-19 (9).

En suma, la APS peruana sigue atomizada o fragmentada, a cargo de diferentes instituciones, sin que sea considerada en modo constante las necesidades de la comunidad, y por eso las políticas sanitarias no dan los resultados esperados (10). Las personas, incluso las que tienen los menores ingresos económicos, deben invertir lo poco que tienen, en análisis, radiografías y cirugías, servicios que, desgraciadamente, el Sistema de Salud no es capaz de proveer, por lo que se requieren políticas financieras más agresivas de parte del Estado para poder proteger a los grupos más vulnerables de nuestra población (11). La integralidad no es precisamente una de las virtudes de nuestro sistema de APS, pese a que desde el 2011 está vigente el Modelo de Atención Integral en Salud Basado en Familia y Comunidad (MAIS-BFC), que, de acuerdo lo plasmado, debería tomar en cuenta las necesidades de salud, expectativas y demandas del individuo, de manera que las decisiones en relación a la salud de las personas sean compartidas ${ }^{(10)}$.

Finalmente, pese a que tenemos una Norma Técnica del Sistema de Referencia y Contrarreferencia de los Establecimientos del Ministerio de Salud que sigue vigente ${ }^{(12)}$, es evidente la falta de una adecuada coordinación entre los diferentes Niveles de Atención que garantice la continuidad de la atención en salud; y es que los distintos niveles tienen deficiencias en su desarrollo, nivel tecnológico, aprovechamiento de la tecnología ${ }^{(10)}$. Todas estas carencias y dificultades se han hecho patentes en el presente panorama desolador de la covid-19 y han contribuido de alguna manera a enfrentar con menos eficiencia la carga de enfermedad por el SARSCoV-2 y en tal sentido los autores nos preguntamos si acaso está faltando coordinación activa al interior del Consejo Nacional de Salud que no solo incorpora instituciones como el Minsa, EsSalud, sino además a la Superintendencia Nacional de Educación Universitaria, Colegio Médico Sanidades de las Fuerzas Armadas y Policiales, Instituto Nacional Penitenciario (INPE) y representantes de la sociedad civil, de tal manera que la coordinación intersectorial en el ámbito de la Salud debiera darse a un nivel que la salud y seguridad de las personas debiera estar garantizada ${ }^{(13)}$.
Inversión pública reducida en el sector salud. Pese al crecimiento económico del Perú en los últimos 15 años, en el año 2007, el Perú fue el país con menor porcentaje de gasto público en salud respecto al producto bruto interno (PBI), y para el 2014, el Perú fue el segundo país de América Latina con el menor porcentaje del PBI destinado a salud (10). De hecho, según aproximaciones publicadas, en el 2017 el gasto en salud en el Perú representó el 3,17\% del PBI, y de 3,18\% en el $2016{ }^{(14)}$. De igual manera, para el 2017, el Perú había dedicado el $14,9 \%$ de su gasto público en salud ${ }^{(14)}$.

Por otro lado, no se trata solamente de cantidad de dinero invertido en salud, sino que se debe analizar la eficiencia y efectividad, determinar si el dinero destinado sirvió para construir nuevos establecimientos en los lugares donde era más necesario, de acuerdo a los quintiles de pobreza ${ }^{(15)}$. En efecto, la inversión en salud tendría que estar orientada a cerrar las brechas de oferta aún existentes, en especial en las personas con pobreza y extrema pobreza.

Los lados positivos de la inversión en salud son, por ejemplo, Perú aumentó el gasto per cápita de US\$ 359 en 2014 a US\$ 626 en 2016; sin embargo, eso no ha servido aparentemente mucho porque en el 2007 Perú ocupaba el último lugar en la región y en el 2014 pasó a ocupar el penúltimo lugar en términos de porcentaje del $\mathrm{PBI}$ destinado para salud ${ }^{\left({ }^{16}\right)}$.

Según cifras del Banco Mundial, el Perú aún se mantiene rezagado respecto a los demás países de América Latina pese a que el presupuesto asignado al sector Salud se incrementó un $63,4 \%$ en los últimos cinco años, y que el gasto público ejecutado llegó a un $60,7 \%$ (17). A excepción de México, nuestro país había invertido menos de su PBI en salud que el resto de países de la Alianza del Pacífico ${ }^{(17)}$.

El otro lado que se debe analizar es la capacidad de gasto de los gestores regionales en salud, situación que se ha hecho más patente en plena pandemia. Por ejemplo, hacia mayo del año 2019 , la ejecución de gasto del presupuesto en salud era de $32,3 \%$, mientras que el de los gobiernos locales era en promedio de $29,9 \%$ (17). Y en este momento, mientras siguen muriendo personas infectadas, la región Cajamarca tiene $48 \%$ de ejecución de gasto, Arequipa, $68,1 \%$, Loreto en $42,3 \%$ (18).

Hay que reconocer, sin embargo, que el Gobierno hace sus mejores esfuerzos para asignar fondos destinados al SIS en el marco del objetivo de aseguramiento universal. Para este año, el Estado decidió financiar al SIS con 1600 millones de soles, tanto para los establecimientos de Lima como para las 25 regiones del Perú, benefician- 
do a más de 20 millones de afiliados ${ }^{(19)}$.

En suma, pese a los esfuerzos del Gobierno, los fondos económicos asignados no parecen ser suficientes, porque el gasto de bolsillo de hogares sigue siendo preponderante para recuperar o mantener la salud; ese gasto en salud ha ido disminuyendo con el tiempo, a medida que el Estado ha inyectado más fondos en el Sector, pero aún es alto, a pesar de que, por ejemplo, en 2008 tenía una participación de 41\% en el gasto total en salud y disminuyó a 29\% en $2014{ }^{\left({ }^{16}\right)}$.

La corrupción en el Perú se comporta esencialmente en la modalidad de aprovechamiento personal o colectivo de los fondos públicos, y ha aumentado en relación directamente proporcional a la mejora del estado económico peruano en las últimas décadas; de hecho, un interesante y revelador artículo publicado en The Lancet señala que la corrupción puede tomar diversas modalidades como ejemplo nepotismo, tráfico de influencias, desfalco, ausentismo laboral, registro de procedimientos que nunca fueron ejecutados o no bien sustentados ${ }^{(21)}$.

La corrupción en el Perú se comporta esencialmente en la modalidad de aprovechamiento personal o colectivo de los fondos públicos, y ha aumentado en relación directamente proporcional a la mejora del estado económico peruano en las últimas décadas; de hecho, un interesante y revelador artículo publicado en The Lancet señala que la corrupción puede tomar diversas modalidades como ejemplo nepotismo, tráfico de influencias, desfalco, ausentismo laboral, registro de procedimientos que nunca fueron ejecutados o no bien sustentados ${ }^{(21)}$.

En plena pandemia, la corrupción tomó varias formas, por ejemplo, compras sobrevaloradas de insumos y equipos necesarios para la prevención y manejo de la covid-19, casos que motivaron sanciones y el inicio de investigaciones al interior de la Policía Nacional del Perú (PNP) ${ }^{(22)}$. No solo se descubrió sobrevaloración, sino que, por ejemplo, una empresa que le vendió insumos a la PNP era conducida por la esposa de un oficial de alto rango y también se presume que se compraron productos no aptos para el consumo humano (22).

La falta de apoyo para la gestión de la producción científica y tecnología en los servicios de salud. Un sector de tanta trascendencia como Salud necesita del apoyo de otros actores o instituciones para disponer de conocimiento producto de la investigación científica y tecnológica, con la finalidad de otorgar mejores servicios, incluso a las personas que viven en los lugares más alejados del Perú.

La coordinación entre el sector salud y universidades, por ejemplo, no ha tenido la fluidez necesaria, pero hay que reconocer que los esfuerzos ya empezaron, aunque es preciso señalar que las universidades tampoco han mostrado entusiasmo en usar fondos para generar conocimiento en bien de la comunidad, salvo honrosas excepciones, por lo que se requiere definir estrategias para modificar la situación, tomando en cuenta que ciertas regiones del Perú acceden a dinero procedente del canon minero y urge generar investigación científica y tecnología en beneficio de sus respectivas comunidades ${ }^{233)}$. Debemos reconocer en este punto que Concytec y el Instituto Nacional de Salud (INS) juegan un rol esencial en la orientación y la canalización de los fondos destinados a financiar el desarrollo de proyectos de investigación orientados a generar nuevo conocimiento para optimizar la prevención, diagnóstico, manejo y rehabilitación en el caso de las prioridades nacionales de salud, y en este momento con extremo interés en la covid-19 ${ }^{(24)}$.

En resumen, el Estado ya está tratando de superar el letargo de décadas de abandono y una de las mejores expresiones para cerrar las brechas en la atención es precisamente la Telemedicina. En ese sentido, el Gobierno mediante Ley 30421 y su Reglamento ha impulsado el uso de herramientas de las Tecnologías de Información y Comunicación (TIC), Ley Marco de Telesalud, y de esa manera se ha consolidado estrategias como la teleconsulta, teleorientación, telemonitoreo, herramientas que cobran relevancia en medio de la pandemia, pero también para definir la conducta a seguir en el caso de patologías complejas como las neoplasias sólidas o hematológicas ${ }^{25)}$.

CONCLUCIONES: A pesar del crecimiento económico del Perú, continúan las inequidades en el acceso a los servicios de salud, situaciones que empeoran por la actividad de redes de corrupción. Es importante fortalecer el Primer Nivel de Atención, justo ahora, en plena Pandemia, incorporándolo a la red de Telesalud que ha demostrado su eficacia en llegar hasta lugares muy alejados del país con soluciones inmediatas gracias a las bondades de las TIC.

Conflictos de interés: Los autores declaran no tener conflictos de interés en esta publicación.

\section{Fuentes de financiamiento: Autofinanciado}

\section{REFERENCIAS BIBLIOGRÁFICAS}

1. Comisión Económica para América y el Caribe. Impactos de la pandemia en los sectores productivos más afectados abarcarán a un tercio del empleo y un cuarto del PIB de la región. [Internet]. Santiago de Chile.www.cepal.org. [citado el 26 de julio de 2020].https://www. cepal.org/es/comunicados/impactos-la-pandemia-sectores-productivos-mas-afectados-abarcaran-un-tercio-empleo-un

2. Horowitz J. El sistema de salud de Italia sufre por el coronavirus: una advertencia para el mundo. [Internet]. The New York Times. 16 de marzo 2020. [citado el 31 de agosto de 2020]. https://www.infobae.com/america/the-new-york-times/2020/03/16/el-sistema-de-salud-de-italia-sufre-por-el-coronavirus-una-advertencia-para-el-mun$\mathrm{do} /$

3. Ruiz A. Estados Unidos superó los cinco millones de contagios [Internet]. France 24. 8 de agosto 2020. [citado el 31 de agosto de 2020]. https://www.france24.com/es/20200808-covid19-eeuu-contagios-muertes-virus

4. Alcalde-Rabanal J, Lazo-Gonzales O, Macias N, Contreras-Ochoa C, 
Espinosa-Henao O. Sistema de salud de Perú: Situación actual, desafíos y perspectivas. Revista Internacional de Salud Materno Fetal. 2019; 4(3): $8-18$.

5. Diario El Comercio. ¿Qué clínicas firmaron contrato con el SIS para atender a pacientes COVID-19? Revisa aquí la lista completa. [Internet] Lima.[citado el 26 de julio de 2020].Disponible en : https://elcomercio. pe/respuestas/que-clinicas-firmaron-contrato-con-el-sis-para-atendera-pacientes-covid-19-revisa-aqui-la-lista-completa-minsa-clinicas-privadas-sis-usi-atencion-medica-pacientes-internacional-anglo-americana-san-pablo-stella-maris-coronavirus-peru-pandemia-revtli-noticia/

6. EsSalud. El Sistema de Salud del Perú: situación actual y estrategias para orientar la extensión de la cobertura contributiva. [Internet]Lima. [citado el 27 de julio de 2020]. Disponible en: http://bvs.minsa.gob.pe/local/ MINSA/2401.pdf

7. Sánchez-Moreno F. El sistema nacional de salud en el Perú. Rev Peru Med Exp Salud Publica. 2014;31(4): 747-53.

8. The Pan American Health Organization Promoting Health in the Americas. DECLARACION DE ALMA-ATA. [Internet].[citado el 27 de julio de 2020]. Disponible en: https://www.paho.org/hq/dmdocuments/2012/Alma-Ata-1978Declaracion.pdf

9. Diario Gestión. Un grito de auxilio en la Amazonía. [Internet] .[citado el 28 de julio de 2020].Disponible en : https://gestion.pe/peru/coronavirus-un-grito-de-auxilio-en-la-amazonia-iquitos-noticia/

10. Cuba-Fuentes M, Romero Albino Z, Domínguez R, Rojas L, Villanueva R. Dimensiones claves para fortalecer la atención primaria en el Perú a cuarenta años de Alma Ata. An Fac med. 2018:79(4): 346-50. DOI: http:/l dx.doi.org/10.15381/anales. v79i4.15642

11. Pavone MP, Sánchez EJ. Determinantes del gasto de bolsillo en salud de la población pobre atendida en servicios de salud públicos en Perú, 2010-2014 Rev Panam Salud Publica. 2018;42(1): pp.1-7. doi:10.26633/ RPSP.2018.20

12. Ministerio de Salud. Norma técnica del sistema de referencia y contrarreferencia de los establecimientos del Ministerio de Salud (N T $\mathrm{N}^{\circ}$ 018-MINSA/DGSP-V.01). [Internet]. [citado el 28 de julio de 2020]. Disponible en: https://www.gob.pe/institucion/minsa/informes-publicaciones/352888-norma-tecnica-del-sistema-de-referencia-y-contrarreferencia-de-los-establecimientos-del-ministerio-de-salud-n-t-n-018-minsa-dgsp-v-01

13. Ministerio de Salud. Consejo Nacional de Salud. [Internet].[citado el 28 de julio de 2020]. Disponible en: https://www.gob.pe/7890-consejo-nacional-de-salud

14. Datosmacro.com. Perú - Gasto público Salud.[Internet].[citado el 28 de julio de 2020]. Disponible en: https://datosmacro.expansion.com/estado/ gasto/salud/peru

15. Huayanay-Espinoza CA, Huayanay A, Huicho L. Incremento en el gasto por inversión en el sector salud: ¿eficiencia y efectividad en el gasto? [carta]. Rev Peru Med Exp Salud Publica. 2015;32(4):822-4.

16. Esan.edu.pe. El financiamiento de la salud en Perú. [Internet]. Lima.[citado el 28 de julio de 2020]. Disponible en: https://www.esan.edu.pe/apuntes-empresariales/2017/04/el-financiamiento-de-la-salud-en-peru/

17. Diario Gestión. Comex: Perú gasta en salud por debajo del promedio en América Latina. [Internet]. Lima. [citado el 28 de julio de 2020]. Disponible en: https://gestion.pe/economia/comex-peru-gasta-salud-debajo-promedio-america-latina-268172-noticial

18. Ministerio de Salud. Ejecución Presupuestal Covid-19 Perú Regional. [Internet]. Lima. [citado el 28 de julio de 2020]. Disponible en: https://www. minsa.gob.pe/reunis/data/covid19_ejecucion.asp

19. Andina.pe. SIS transfiere cerca de $S /$ 1,000 millones para garantizar cobertura de afiliados en 2020. [Internet]. Lima. [citado el 28 de julio de 2020]. Disponible en: https://andina.pe/agencia/noticia-sis-transfiere-cerca-s-1000-millones-para-garantizar-cobertura-afiliados-2020-786892. aspx

20. Grupo de Investigación en Gobierno, Administración y Políticas Públicas. Corrupción en el sector salud: Tipologías de prácticas de corrupción en los servicios de salud en Lima, Perú. [Internet]. Lima. [citado el 28 de julio de 2020]. Disponible en: http://www.gigapp.org/index.php/mis-publicaciones-gigapp/publication/show/2873

21. García PJ. Corruption in global health: the open secret. Lancet. 2019;394(10214): 2119-24. doi: 10.1016/S0140-6736(19)32527-9.

22. Diario Gestión. Fiscalía investiga 15 denuncias a nivel nacional por compras sobrevaloradas para PNP. [Internet]. Lima. [citado el 28 de julio de 2020]. Disponible en: https://gestion.pe/peru/politica/coronavirus-peru-fiscalia-investiga-15-denuncias-a-nivel-nacional-por-compras-sobrevaloradas-para-policia-nacional-pnp-covid-19-nndc-noticia/
23. Romaní F, Cabezas C, Espinoza M, Minaya G, Huaripata J, Ureta JM, et al. Estrategia para fortalecer las capacidades de investigación en salud en universidades públicas regionales: rol del canon y del Instituto Nacional de Salud. Rev Peru Med Exp Salud Publica. 2012;29(4): 54954.

24. Instituto Nacional de Salud. Convocatoria Proyectos de Investigación COVID-19: INS 2020-I. [Internet]. Lima. [citado el 28 de julio de 2020]. Disponible en: https://web.ins.gob.pe/es/investigacion-en-salud/financiamiento/convocatoria-investigacion-covid19-ins-2020

25. Diario El Peruano. 2 de abril, 2016 [Internet]. Lima. [citado el 28 de julio de 2020]. Disponible en : http://www.leyes.congreso.gob.pe/Documentos/Leyes/30421.pdf 